\title{
Le jaguar, sujet et objet du sacrifice maya
}

\section{Claude-François Baudez}

\section{OpenEdition}

Journals

Édition électronique

URL : https://journals.openedition.org/jsa/9513

DOI : 10.4000/jsa.9513

ISSN : 1957-7842

Éditeur

Société des américanistes

Édition imprimée

Date de publication : 15 juillet 2008

Pagination : 177-189

ISSN : 0037-9174

\section{Référence électronique}

Claude-François Baudez, « Le jaguar, sujet et objet du sacrifice maya », Journal de la Société des américanistes [En ligne], 94-1 | 2008, mis en ligne le 10 juin 2013, consulté le 03 septembre 2022. URL http://journals.openedition.org/jsa/9513 ; DOl : https://doi.org/10.4000/jsa.9513 


\title{
LE JAGUAR, SUJET ET OBJET DU SACRIFICE MAYA
}

\author{
Claude-François BAUDEZ *
}

\begin{abstract}
Dans l'iconographie maya, le jaguar est bien connu dans son rôle de prédateur associé à toutes sortes de violence, qu'il s'agisse de guerre, de sacrifice, de torture ou d'autosacrifice. Le félin apparaît aussi - bien que plus discrètement - dans le rôle symétrique et inverse de victime, que ce soit d'une mise à mort sur l'autel ou de la torture sur l'échafaud. Identifié au guerrier, dont le destin est de vaincre et d'être vaincu, le jaguar est à la fois sacrifiant et sacrifié. [Mots-clés : Maya, jaguar, sacrifice, autosacrifice.]
\end{abstract}

El jaguar, sujeto y objeto del sacrificio maya. En la iconografia maya, el papel agresivo del jaguar, asociado a todas clases de violencia tales como guerra, sacrificio, tortura o autosacrificio, es bien conocido. Hay que recordar, sin embargo, que el felino también aparece en el papel simétrico e inverso de víctima, ya sea en el altar sacrificial o en el cadalso. Identificado con el guerrero, cuyo destino es vencer y ser vencido, el jaguar es al mismo tiempo sacrificador y sacrificado. [Palabras claves : maya, jaguar, sacrificio, autosacrificio.]

The jaguar, subject and object in Maya sacrifice. In Maya iconography, the role of the jaguar as a predator associated with all kinds of violence such as war, sacrifice, torture and self-sacrifice, is well-known. Less obvious is its symmetrical and inverse role of victim, on the altar or on the scaffold. Likened to the human warrior, whose fate is to defeat and be defeated, the jaguar is both sacrificer and sacrified. [Key words: Maya, jaguar, sacrifice, autosacrifice.]

Cette contribution se propose de souligner la double vocation, de sacrificateur et de victime, du jaguar dans le sacrifice maya. L'image emblématique du félin, présenté tantôt comme sujet (sacrificateur ou bourreau), tantôt comme objet (victime), permet de mieux comprendre la conception maya du sacrifice, dans laquelle les deux rôles sont interchangeables.

* Archéologue, directeur de recherche honoraire au CNRS [claude.baudez@orange.fr].

Journal de la Société des Américanistes, 2008, 94-1, pp. 177-189. O Société des Américanistes. 


\section{LE JAGUAR BOURREAU}

L'iconographie de l'art maya montre le jaguar étroitement associé à la guerre et au sacrifice, à l'inframonde et à la mort. Il est l'emblème du soleil nocturne dans son voyage souterrain d'ouest en est, opposé et complémentaire de l'oiseau aux vives couleurs (quetzal, perroquet) qui représente le soleil diurne. Dans les mythes comme dans les images, une plus grande place est accordée au soleil de la nuit et au jaguar plutôt qu'au soleil diurne et à l'oiseau. Le plus grand prédateur de la faune mésoaméricaine, qui ne craint personne sinon l'homme, est naturellement l'emblème privilégié de la royauté et de la noblesse mayas. Les grands utilisent son image dans leur costume (heaume de jaguar, ou jupe en peau du même animal) et dans les objets qui les entourent. Ils peuvent brandir un sceptre en forme de patte griffue, ou être assis sur un trône en forme de félin, souvent bicéphale. L'imagerie guerrière, comme on peut la voir dans la deuxième pièce de la Structure 1 de Bonampak, fait constamment appel au félin.

Sur les vases, si le jaguar n'est pas l'exécuteur direct d'un sacrifice humain, il lui arrive de tenir une tête humaine entre ses pattes (K5074) ${ }^{1}$ ou de présenter; seul ou en compagnie d'autres animaux, des parties de corps humain, comme des mains ou des yeux (K1380) dans un bol. Le plus souvent cependant, son rôle est d'assister à, ou même de présider, une scène sacrificielle, en se joignant aux acteurs de la scène, souvent illustrée, dans laquelle un sacrificateur danse devant sa victime, à laquelle un squelette tend les bras (Figure 1 [K1003] ; K2208, K1256, K2802, K928). Dans ces exemples, il n'est apparemment engagé dans aucune action. Debout ou à quatre pattes, il porte un foulard autour du cou et parfois un serpent est enroulé autour de son corps.

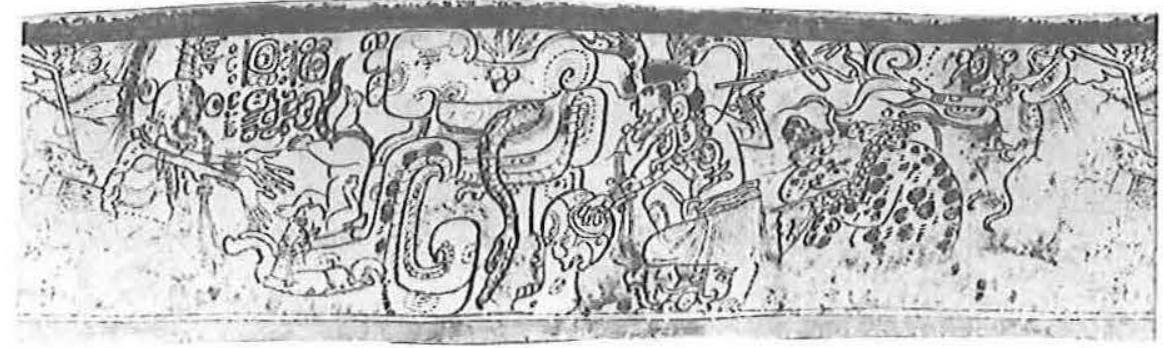

Fig. 1 - Scène de sacrifice à laquelle participe un jaguar, du côté des bourreaux. La victime au corps humain, mais à la queue féline, tombe la tête la première, sur le museau d'un masque cauac qui sert d'autel (d'après Robicsek et Hales 1981, vessel 19). 
Même sans représentation explicite de sacrifice, le jaguar est souvent montré en compagnie d'un squelette (K1652, K1380, K1973). Des animaux comme le cerf (K3043, K5017, K3312, K8733, K3060) - auquel il emprunte souvent un bois pour souligner les liens symboliques qu'il entretient avec cet animal-, le crapaud (K531, K1442), le singe (K3038, K8733, K3060, K5070) ou le dindon (K3470), peuvent se joindre à lui dans des scènes que l'on situe dans l'inframonde. Sur le vase K1230, le jaguar accompagne une scène d'auto-décapitation.

Le félin est aussi très proche du bourreau qui torture. Les instruments de supplice ou qui font allusion à ce rituel sont les couteaux de silex ou d'obsidienne, dits excentriques, et, en particulier, la pierre taillée en trident qui représenterait les griffes du félin. Déjà une peinture olmèque de la grotte de Juxtlahuaca montrait un personnage habillé en jaguar tenant un captif au bout d'une corde et un instrument de type trident (Gay 1967). Sur le linteau 2 du temple III de Tikal (Jones et Satterthwaite 1982, fig. 72), le souverain, vêtu en jaguar, et ses deux acolytes tiennent en main bâton et trident. Si cette scène représente une scène de torture, et cela bien qu'aucune victime ne soit montrée, elle évoque un des épisodes du « sacrifice gladiateur 》 aztèque et mixtèque, illustré sur la page $83 \mathrm{du}$ codex Nuttall, qui montre le tlahuahuanaliztli ou l'action de rayer, de griffer une victime, exécutée par des guerriers-jaguars munis de gants griffius (Nuttall 1975). Dans les mêmes cultures, le sacrifice par flèches (où la victime est identifiée à un gibier ?), dit tlacacaualiztli, est une autre torture, exécutée le jour de la fête dite " écorchement des hommes » célébrée sous le patronage de Xipe Totec. Dans les deux cas, la victime exprime sa douleur par des pleurs.

Sur un vase maya du Classique Récent conservé au Kimbell Art Museum, le personnage qui marche derrière un captif mis à nu porte un jaguar tête en bas ; il brandit un couteau excentrique emmanché, prêt à torturer sa victime (Schele et Miller 1986, pl. 84). Un autre vase montre le jaguar associé à la fois au sacrifice humain et à la torture (Figure 2); on y voit le félin dansant, en compagnie d'un sacrificateur armé de la hache et de la pierre tronée sculptée en forme de tête humaine et d'un squelette armé du trident et de la version maya du bâton-hochet ; à leurs pieds, une tête coupée gît sur le sol, et d'autres restes humains sont rassemblés dans un bol. L'animal a le corps entouré de nénuphars et d'autres symboles aquatiques, montrant ainsi que la vie et la fertilité sont inséparables du sacrifice et de la mort.

Deux aspects emblématiques du félin revêtent une importance particulière dans l'iconographie de la sculpture classique. Celui que l'on appelle dans la littérature, le " jaguar de l'inframonde », apparaît comme un patron de la guerre (Figure 3a-c). Son visage a pour traits caractéristiques deux cordons qui soulignent les yeux et se rejoignent en torsade au-dessus du nez; des oreilles de jaguar visibles au-dessus d'ornements de jade ; des grands yeux carrés avec une pupille en crochet ; des incisives taillées en $\mathrm{T}$ et parfois de grandes moustaches qui encadrent la gueule. Il décore le bouclier qui constitue l'icône centrale du 


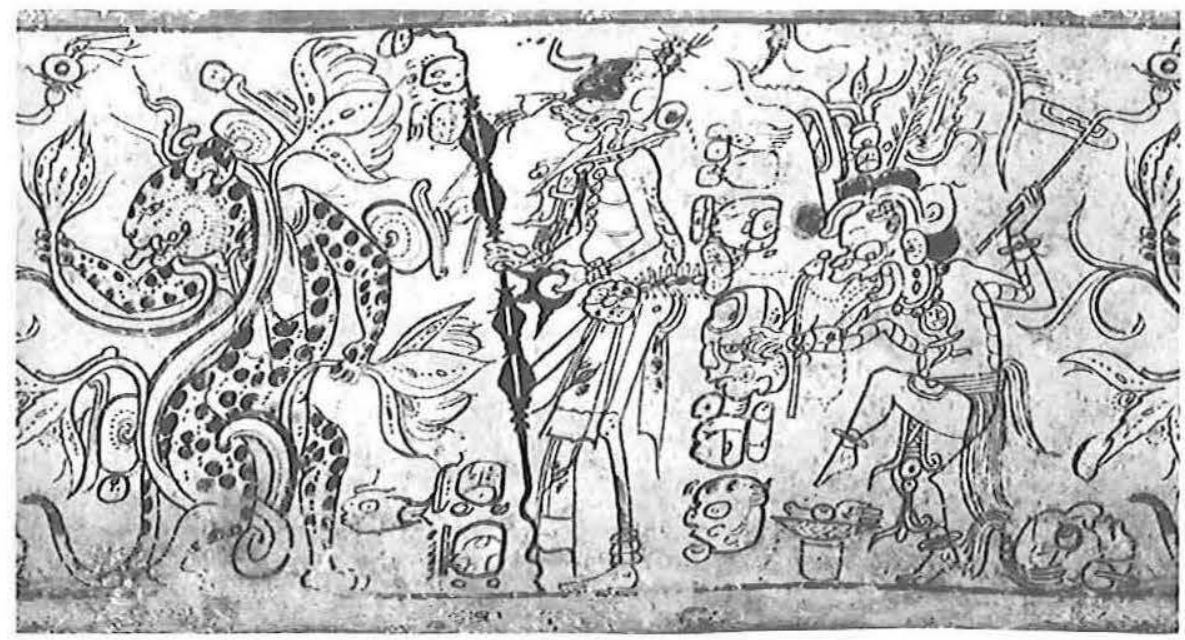

FIG. 2 - Un jaguar, chargé de plantes et de symboles aquatiques, danse en compagnie d'un squelette et d'un sacrificateur (d'après Robicsek et Hales 1981, vessel 30).

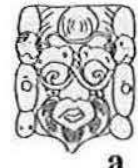

a

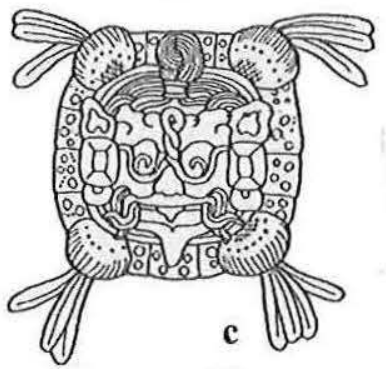

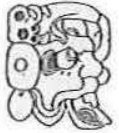
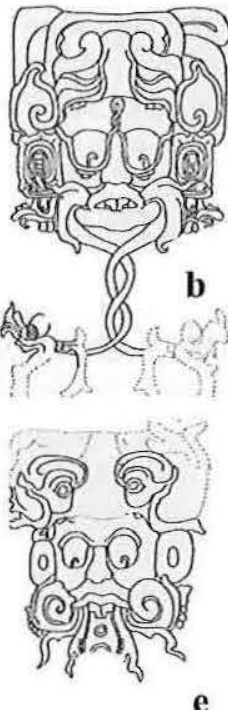

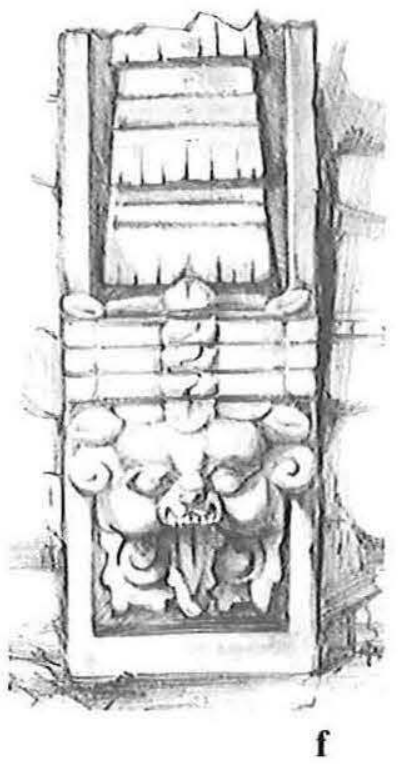

Fig. 3 - Jaguar Uo et Jaguar Pax.

Jaguar Uo ou "Jaguar de l'Inframonde » : a) masque central de ceinture sur la Stèle I de Copan ; b) masque central de ceinture sur la Stèle C Ouest de Copan ; c) bouclier au centre du panneau principal du temple du Soleil de Palenque.

Jaguar Pax : d) masque latéral de ceinture sur la stèle I de Copan ; e) masque latéral de ceinture sur la Stèle C Ouest de Copan ; f) décor en stuc de la rampe ouest de l'escalier nord de la Structure D5-1 de Tonina. 
panneau du temple du Soleil de Palenque (Figure 3c). À Copan, au niveau de la place qui s'étend devant la Structure 24 et qui correspond, dans ce microcosme, au fond des enfers, trois dalles associent des boucliers à une tête de jaguar rugissant (Baudez 1994, pp. 213-216, fig. 106c). Le " jaguar de l'inframonde » existe sous forme glyphique (où il a la valeur du chiffre 7), mais aussi comme emblème de bouclier ou de masque de ceinture. Il est le patron du mois Uo et du jour $A$ kbal. Sur le linteau 2 du temple IV de Tikal (Jones et Satterthwaite 1982, fig. 73), le souverain trônant est protégé par un anthropomorphe de grande taille paré des éléments distinctifs du jaguar $U o$ : chiffre 7 , torsade sur le nez et oreille de jaguar.

Au jaguar Uo s'oppose le jaguar Pax, patron du mois du même nom. Les deux aspects $U o$ et $P a x$ du félin sont réunis, comme masques de ceinture, sur les stèles I et C de Copan (Figure 3a-b, d-e ; Baudez 1994, pp. 33-35, 68-69, fig. 118). Le jaguar Pax, une figure emblématique du sacrifice, n'a pas de mandibule ; ses yeux sont souvent cerclés et des volutes crénelées encadrent sa langue pointue (Figure 3d-f). Celle-ci, aux bords souvent en dents de scie, représente un couteau qui indique la soif de sang de la créature. Une ou trois bandes nouées, insignes du sacrifice, ornent son front. Les traits proprement félins sont les oreilles, parfois remplacées par des pattes griffues, qui dépassent des ornements de jade. L'efligie du jaguar Pax, modelée en stuc, orne les rampes de la Structure D5-1 de Tonina (Figure 3f). La tête d'une statue de jaguar en stuc possédant toutes les caractéristiques de cette créature a été trouvée au pied de la Structure E5-2 du même site (Becquelin et Baudez 1979, p. 48).

Le jaguar Pax se manifeste aussi sur les vases, avec les mêmes traits mais avec un corps humain. Il est assis en compagnie de six autres créatures surnaturelles (K2796), ou bien préside une scène sacrificielle (K1645) auprès d'un autre jaguar (Uo ?).

\section{LE JAGUAR VICTIME}

Le jaguar prédateur, guerrier, sacrificateur ou bourreau a, parfois et même souvent, le rôle inverse de victime. Cependant, dans les tombes et les dépôts de fondation, les restes de félins sacrifiés ne sont pas fréquents, beaucoup moins que d'autres animaux comme les poissons (à Tikal), les animaux amphibies comme le crocodile ou la tortue, ou les oiseaux. Il est néanmoins des cas où l'on peut être certain que le jaguar a été sacrifié en vertu de son rôle symbolique. Ainsi le dépôt IV-1 de Tonina renfermait, outre des squelettes d'enfants sacrifiés et des têtes de cailles, les restes du couple emblématique oiseau de proie/félin (Becquelin et Baudez 1979, p. 173). On retrouve ailleurs en Mésoamérique le sacrifice du jaguar et d'autres animaux choisis en vertu de leur charge symbolique ; notamment dans les dépôts de fondation des diverses étapes de construction de la pyramide de la 
Lune à Teotihuacan (Sugiyama et Cabrera 2003 ; 2005), et dans ceux du grand temple de Tenochtitlan (López Luján 1993).

L'association du jaguar et du pouvoir, évidente dans les images, se manifeste aussi dans l'archéologie. Le dépôt creusé devant l'autel Q de Copan contenait quinze crânes de jaguars décapités (Fash 1991, pp. 169-170) ; ce nombre correspond à celui des prédécesseurs de Soleil Levant, sculptés sur le monument auprès du souverain qui l'a fait ériger. La relation entre le sacrifice des quinze jaguars et l'évocation des quinze rois copanèques est évidente. La décapitation du jaguar dans un contexte royal est illustrée sur le linteau 26 de Yaxchilan (Figure 4). On y voit le roi Bouclier-Jaguar, le couteau de sacrifice à la main, remettant à la reine la tête du félin qu'il vient de sacrifier ${ }^{2}$.

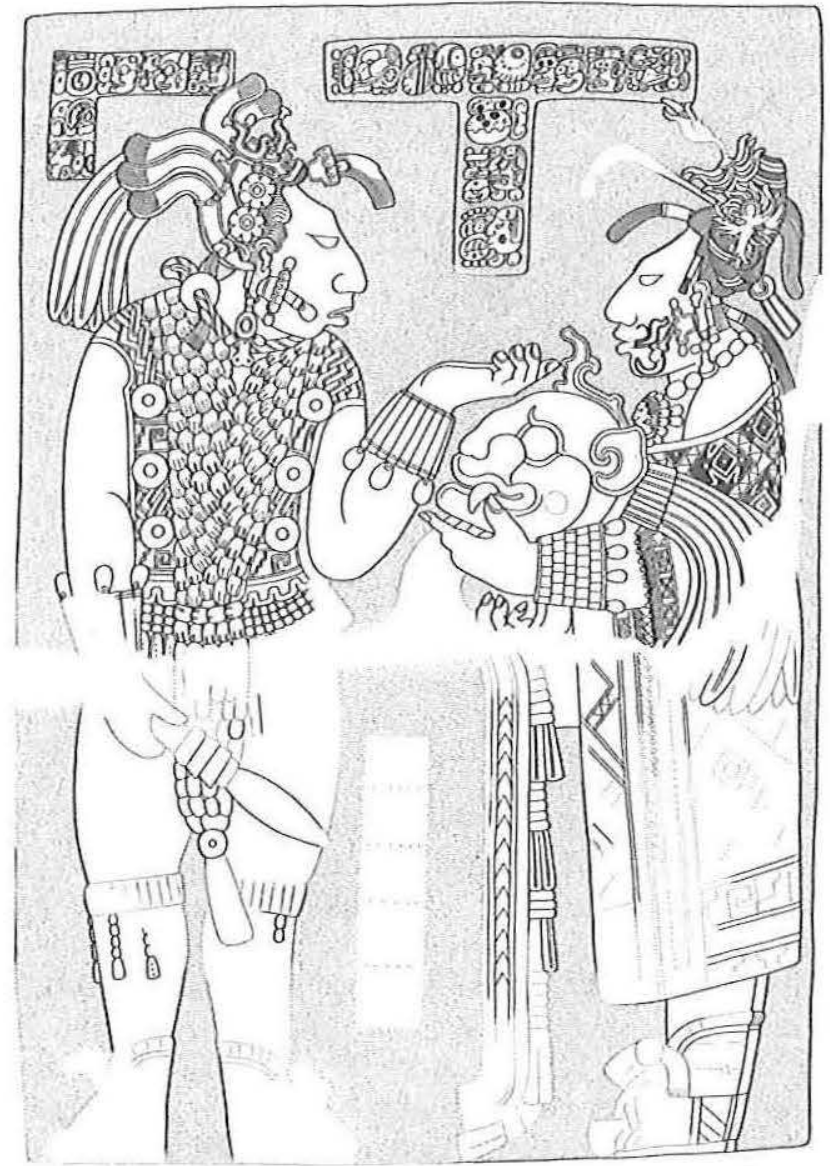

Fig. 4 - Yaxchilan, Linteau 26 : le roi Bouclier-Jaguar remet à la reine la tête d'un jaguar qu'il vient de sacrifier (d'après Graham et von Euw 1977). 
Le sacrifice de félins est souvent traité sur les vases céramiques polychromes. Dans la série de scènes sacrificielles appelée le sacrifice du « jaguar god » (Robicsek et Hales 1981, vessels 17-33), la victime apparaît comme un enfant mi-humain, mi-félin ${ }^{3}$. Rares sont les peintures qui montrent la victime comme un animal sans aucun élément anthropomorphe (Figure 5). Elle gît sur un autel en forme de masque de monstre terrestre cauac ; son bourreau danse en brandissant d'une main une hache et de l'autre une pierre trouée ; un squelette tend les bras vers la victime, comme pour s'en saisir. Des animaux peuvent se joindre à la scène, un jaguar (Figure 1) ${ }^{4}$ ou un insecte et un chien par exemple, comme sur le célèbre vase du Metropolitan Museum de New York (Figure 6). Dans cet exemple, comme sur la majorité des vases de cette série, la victime est un anthropomorphe félinisé. Le corps est celui d'un enfant, la tête est celle d'un adulte, les pattes et la queue sont félines. Sur le vase 26 de la série (Robicsek et Hales 1981), la victime n'a du jaguar que la queue. Ces victimes hybrides sont rarement étendues horizontalement sur le masque qui sert d'autel (Figure 1); le plus souvent, elles tombent à la

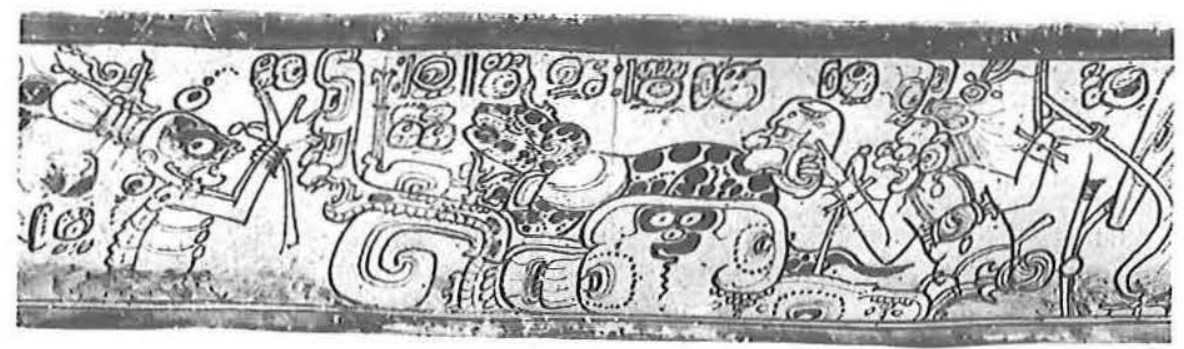

FIG. 5 - Sacrifice d'un jaguar (d'après Robicsek et Hales 1981, vessel 27).

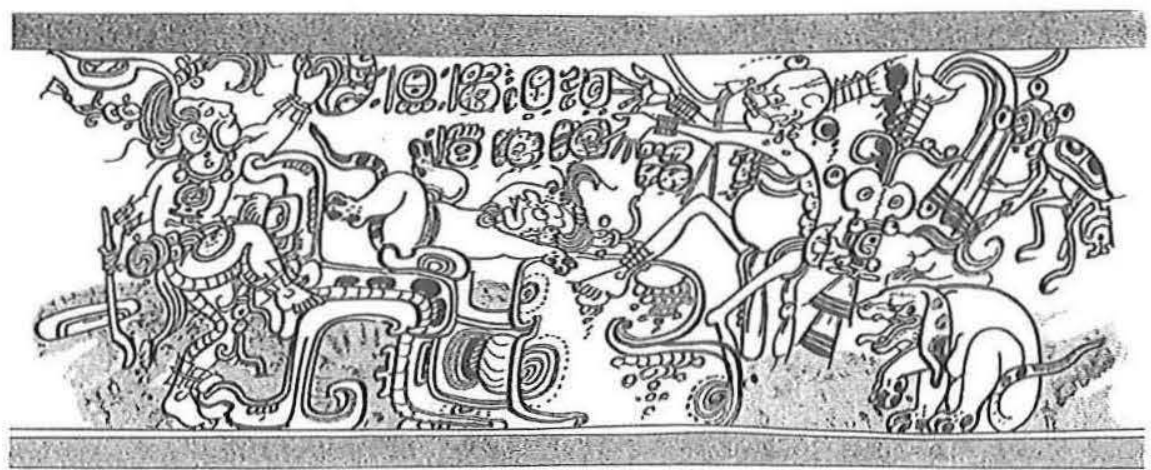

Fig. 6 - Un chien et un insecte assistent à un sacrifice, en compagnie de la Mort qui tend les bras vers la victime, une créature mi-humaine, mi-féline (d'après Coe 1973, vase 45). 

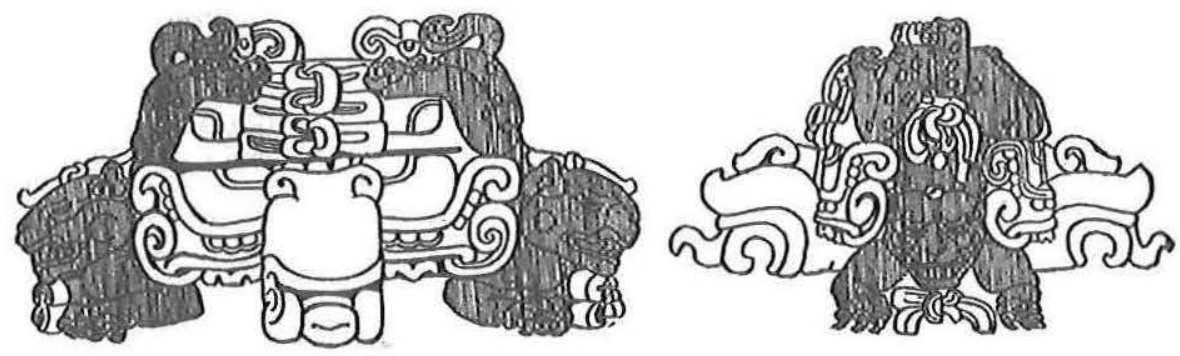

Fig. 7 - L'autel de la Stèle F de Copan réunit les deux rôles du jaguar : le félin prédateur est représenté par les deux têtes de Jaguar Pax dans l'axe est-ouest ; le jaguar victime par les deux félins tombant sur les côtés du monument (d’après Spinden 1913, fig. 99).

renverse, et parfois même la tête la première. Cette posture de chute est souvent adoptée par le félin, comme sur une des images du vase MS 0739 où un jaguar tombe la tête la première sur un trône fait d'os humains (Reents-Budet 1994, p. 273, personage 4) ; on retrouve cette même posture sur les côtés nord et sud de l'autel de la stèle $\mathrm{F}$ de Copan (Figure 7). La chute, cas particulier de l'inversion qui caractérise toute chose « négative », la mort en particulier, réduit le prédateur au rôle de victime.

Après l'exécution, vient l'offrande du sacrifice de la victime mi-humaine, mi-féline. La scène centrale d'un bol polychrome (Robicsek et Hales 1981, fig. 31) montre la victime qui déborde, malgré sa petite taille, du bol dans lequel elle repose (Figure 8). La gueule du serpent qui s'échappe de son corps s'ouvre sur un bouclier, marqué d'une queue de jaguar. Cette allégorie illustre les deux moments du cycle guerre/sacrifice où se trouvent évoqués le jaguar prédateur et le jaguar victime.

Victime de la torture, le jaguar est montré mort ou agonisant (les yeux fermés, il tombe à la renverse) auprès d'un échafaud, sur un vase qui dépeint des danses que l'on situe dans l'inframonde (Figure 9). Un autre vase (K7220) montre aussi un jaguar sur l'échafaud en compagnie d'une victime décapitée et d'un squelette. Un bol provenant de la tombe de la Structure A-1 de Uaxactún présente des bourreaux au corps peint en noir, munis du bâton et de la griffe (Sharer 1994, fig. 15.18) ; leur victime est un jaguar assis qui tient dans ses pattes l'échafaud du supplice. Une autre scène du vase MS 0739, dont le décor est entièrement consacré au sacrifice, la torture du jaguar ou plutôt l'offrande de sa torture, est illustrée par un personnage accroupi qui présente un bâton (réduction d'un échafaud) auquel est attaché un jaguar (Figure 10).

Les félins, victimes de la torture, ont une expression très différente du jaguar qui montre les dents ; ici leur gueule est démesurément béante, comme laissant échapper un rugissement, plus un cri de douleur qu'une expression de menace. 


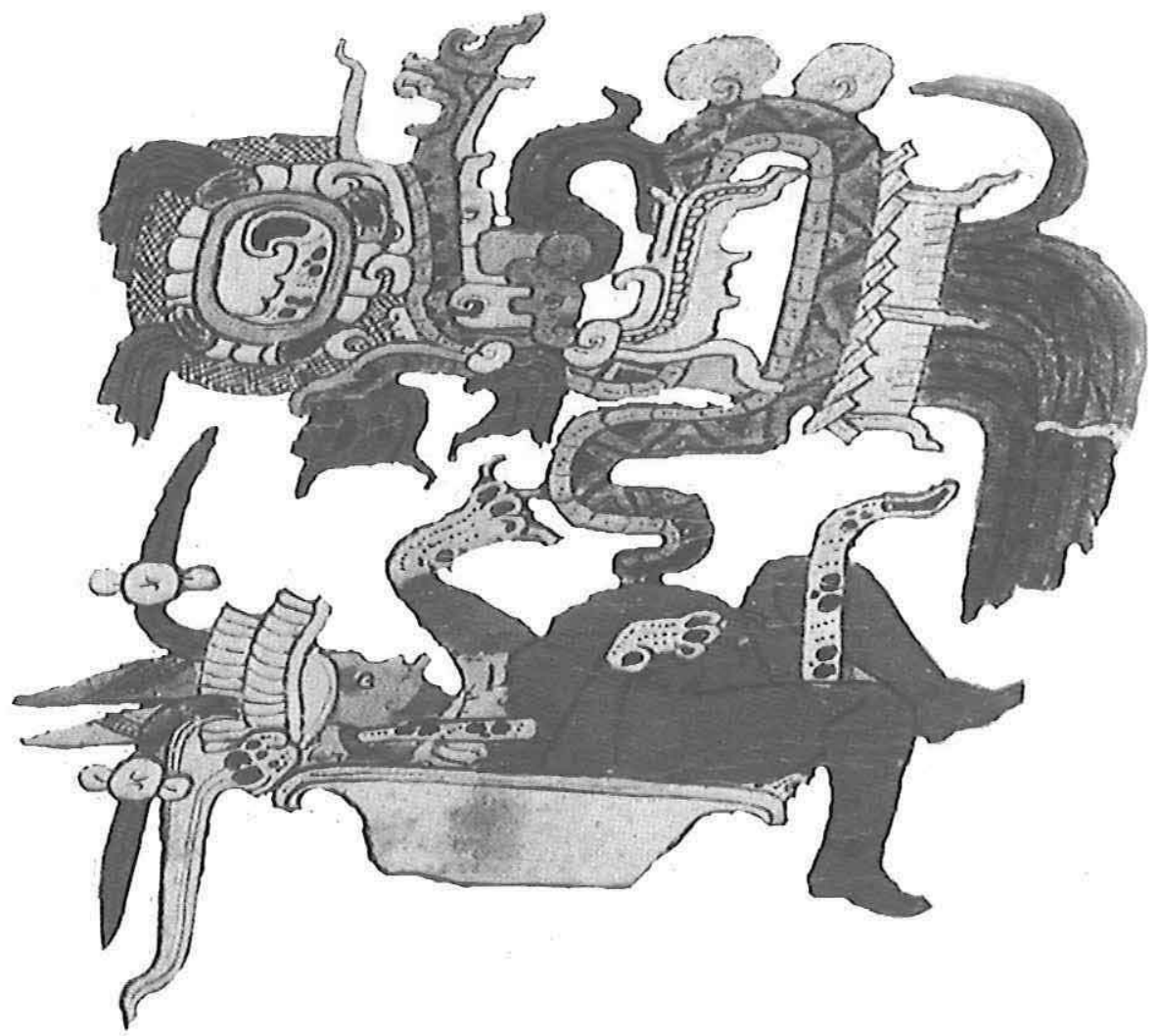

FIG. 8 - Détail d'une scène sacrificielle (d'après Robicsek et Hales 1981, fig. 31).

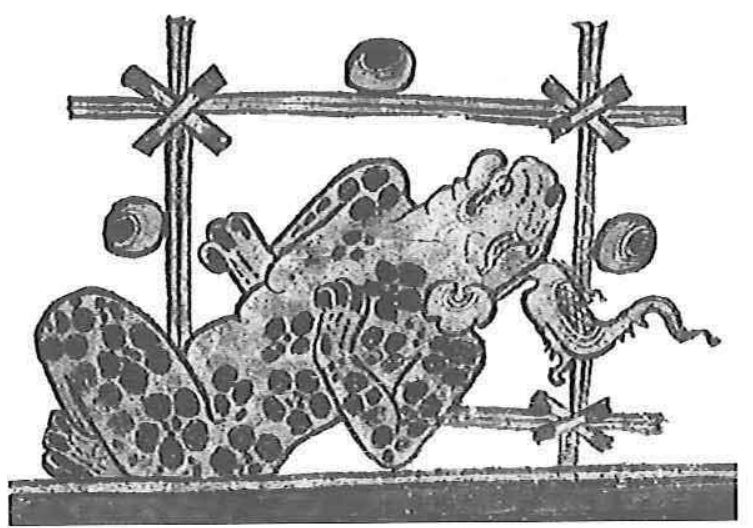

FIG. 9 - Détail d'une scène infernale : jaguar sur l'échafaud (d'après K791). 


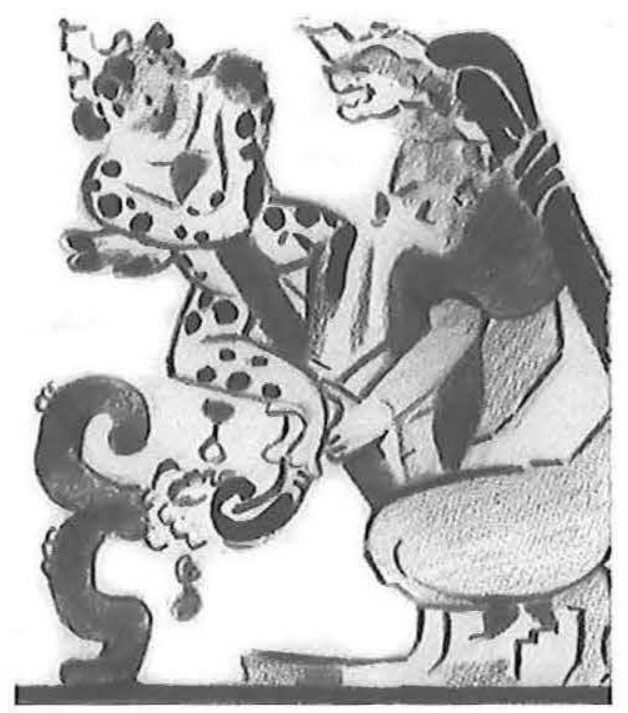

Fig. 10 - Détail - personnage 7-du vase MS 0739: le jaguar attaché à un bâton est l'image en réduction du félin mis torturé sur l'échafaud (aquarelle I. Bonzom, d'après Reents-Budet 1994, p. 273).

Un vase passablement effacé montre un jaguar dans la même attitude qui semble répondre à un homme, en partie costumé en félin, qui présente la même expression d'effroi devant la menace d'une arme pointue (Baudez 2004, fig. 21e).

\section{CONCLuSion}

L'autel de la stèle F de Copan (Figure 7) traduit de façon exemplaire le double rôle du jaguar ; celui de prédateur, de patron du sacrifice, est exprimé sur les faces est et ouest de l'autel, par une tête en haut relief de jaguar Pax (voir plus haut); celui de la victime, par l'animal tombant la tête la première, sur les côtés nord et sud du monument.

L'iconographie cherche parfois dans les relations de guerre, de capture et de sacrifice, à assimiler vainqueur et vaincu, sacrificateur et sacrifié. Si la plupart des représentations cherchent à montrer vainqueur et vaincu aux antipodes l'un de l'autre, il arrive que les vainqueurs portent des insignes que l'on croyait exclusivement réservés aux captifs et, de même, que ceux-ci s'approprient les emblèmes des vainqueurs. Ainsi, sur la stèle 15 de Yaxchilan, le roi Bouclier-Jaguar saisit aux cheveux un captif en même temps qu'il arbore des rubans d'oreille en peau de jaguar, une chevelure attachée ostensiblement sur le sommet du crâne, et une 

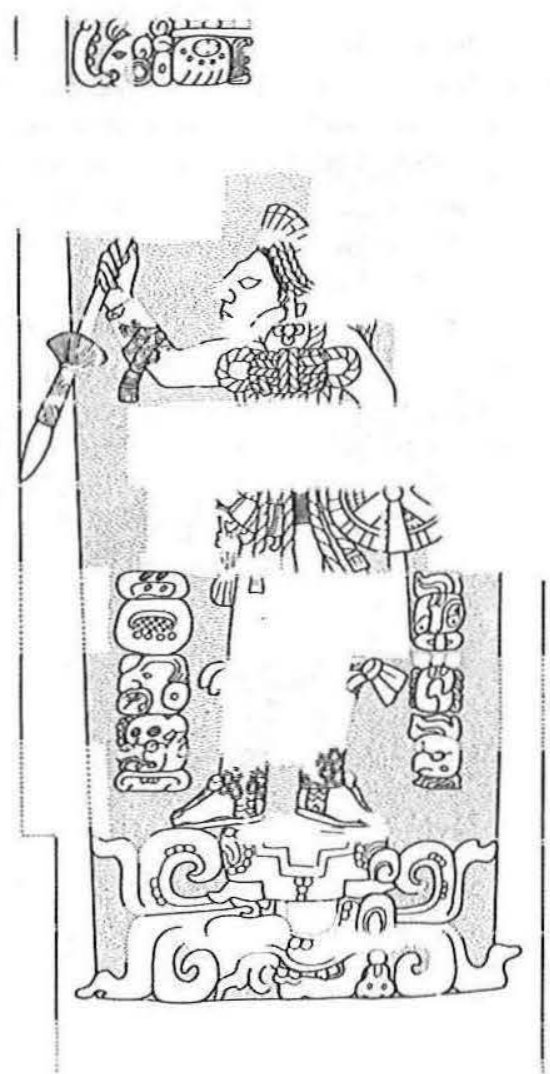

FIG. 11 - Jambage Nord-Est de la Structure 18 de Copan : le roi chargé de cordes s'identifie à son prisonnier (dessin A. Dowd, in Baudez 1994, fig. 95b).

corde nouée sous le genou. Sur un jambage de la Structure 18 de Copan, le roi, armé d'une lance et d'un bouclier, est cependant couvert de cordes (Figure 11). Il arrive aussi que le captif cherche à s'identifier au vainqueur en s'appropriant certains de ses attributs, comme le masque du jaguar de l'Inframonde. La stèle 8 de Naranjo montre le roi portant ce masque, attribut fréquent du pouvoir royal ; or un captif de Tonina, voué au sacrifice, porte le même insigne pour s'identifier au vainqueur (Baudez 2004, fig. 7).

Ces appropriations se font dans les deux sens : le vainqueur s'approprie les rubans d'oreille du vaincu, comme la victime s'approprie le masque de jaguar du vainqueur. L'objectif de ces échanges iconographiques est d'illustrer la profonde identité de nature, sinon de rôles, du guerrier vainqueur et du guerrier vaincu. 
Cette identification du vainqueur et de son captif, du sacrificateur et de sa victime, est démontrée sur la sculpture monumentale maya et elle concerne là des personnages historiques. Sur les vases peints, destinés aux défunts et à leur au-delà, l'allégorie prend le pas sur le monde réel et fait appel au félin pour assumer des rôles opposés et complémentaires et confirmer un aspect fondamental du sacrifice mésoaméricain, bien attesté du moins chez les Aztèques. Chez ces derniers, la confusion des acteurs est apparente grâce à l'emploi des mêmes noms pour désigner des rôles opposés ; par l'établisssement d'une parenté entre eux (le captif appelle " père » son capteur et ce dernier lui répond " mon fils »); par le port, par les uns et les autres, des mêmes costumes et peintures corporelles ; par la participation aux mêmes danses, etc. Dans la fête "Ecorchement des hommes ", l'assimilation prend la forme extrême du sacrifice par l'écorchement des victimes où la peau de ces dernières est revêtue par les guerriers mexicas (Baudez s. d.). *

* Manuscrit reçu en septembre 2006 et accepté pour publication en janvier 2008.

\section{NOTES}

1. Le lecteur est prié de se reporter au Mayavase Database. An archive of rollout photographs created by Justin Kerr auquel il aura accès sur le site de la fondation FAMSI, à l'adresse suivante : http://research.famsi.org/kerrmap.html. Les références sont indiquées, selon l'usage, par un numéro précédé de la lettre K, pour Kerr.

2. L'interprétation courante de ce linteau, due à Peter Mathews (1997), est que la reine remet au roi ses armes - le couteau - et son casque, la tête de jaguar, avant le départ du souverain au combat. Nous contestons cette interprétation parce que le couteau n'est pas une arme de guerre mais de sacrifice, et que le geste du roi envers la reine évoque plus le don que la réception.

3. Comme la victime sacrificielle est presque toujours de petite taille, certains y voient un enfant et interprètent la scène comme un sacrifice d'enfants. Bien que ceux-ci aient effectivement eu lieu, on a plutôt affaire ici à une miniaturisation, où la victime est réduite pour tenir dans un bol. En effet, l'offrande qui suit l'exécution est presque toujours présentée dans un bol qui ne peut contenir la victime qu'à la condition qu'elle soit miniaturisée.

4. Sur ce vase (K 1003), le jaguar est figuré simultanément comme victime (sous l'aspect du « jaguar god ") et comme bourreau ou, plutôt comme il a été dit plus haut, comme assistant de l'exécuteur du sacrifice.

\section{RÉFÉRENCES BIBLIOGRAPHIQUES}

BAudez Claude-François

1994 Maya sculpture of Copan : the iconography, University of Oklahoma Press, Norman.

2004 "Los cautivos Mayas y su destino », in Enrique Nalda (éd.), Los Cautivos de Dzibanché, INAH, Mexico.

s. d. "Sacrificio de si, sacrificio del otro », seminario : Nuevas perspectivas sobre el sacrificio humano entre los mexicas, Mexico [à paraître]. 
BecQuelin Pierre et Claude-François Baudez

1979 Tonina, une cité maya du Chiapas, Mission archéologique et ethnologique française au Mexique, coll. " Études mésoaméricaines » 6-3/Éditions Recherche sur les civilisations, Paris/Mexico.

FAsH William L.

1991 Scribes, warriors and kings. The city of Copán and the Ancient Maya, Thames and Hudson, Londres.

Gay Carlo

1967 "Oldest paintings of the New World », Natural History, 76 (4), pp. 28-35.

Graham Ian et Eric von Euw

1977 Corpus of Maya hieroglyphic inscriptions. Vol. 3, part 1 : Yaxchilan, Peabody Museum, Harvard University, Cambridge.

JoNES Christopher et Linton SatterthwaIte Jr.

1982 The monuments and inscriptions of Tikal: the carved monuments, Tikal Reports 33A, University Museum, University Museum Monographs 44, Philadelphie.

\section{LÓPEZ LUJÁN Leonardo}

1993 Las ofrendas del Templo Mayor de Tenochtitlan, Instituto Nacional de Antropología e Historia, Mexico.

Mathews Peter

1997 La escultura de Yaxchilan, INAH, Mexico.

NutTall Zelia (éd.)

1975 The Codex Nuttall. A picture manuscript from Ancient Mexico, with new introductory text by Arthur G. Miller, Dover Publications, New York.

REENTS-Budet Dorie

1994 Painting the Maya universe: royal ceramics of the Classic Period, Duke University Press, Durham/Londres.

Robicsek Francis et Donald M. HALES

1981 The Maya book of the dead. The Ceramic Codex, The University of Virginia Art Museum, Charlottesville.

SCHELE Linda et Mary Ellen MiLleR

1986 The Blood of Kings. Dynasty and ritual in Maya art, Kimbell Art Museum, New York/Fort Worth.

\section{SHARER Robert J.}

1994 The Ancient Maya, Stanford University Press, Stanford [5th edition].

Sugiyama Saburo et Rubén Cabrera

2003 " Hallazgos recientes en la Pirámide de la Luna », Arqueología Mexicana, 64, pp. 42-49.

2005 "Se localiza otra insólita ofrenda en la Pirámide de la Luna », Arqueología Mexicana, 71, pp, 12-13. 